\title{
Educación para la Ciudadanía Global Crítica al acabar la educación obligatoria en España y Portugal: Una revisión integrativa de la literatura
}

Noelia Santamaría-Cárdaba - Universidad Internacional Isabel I

Amanda Franco - Instituto Politécnico de Viseu

Mónica Lourenço - Universidade de Aveiro

Rui Marques Vieira - Universidade de Aveiro
(iD) $0000-0001-6864-9330$

(D) $0000-0002-7758-4257$

(i) $0000-0002-8124-2452$

(D) 0000-0003-0610-6896

Recepción: 26-01-2022 | Aceptado: 31.01.2022

Correspondencia a través de ORCID: Noelia Santamaría-Cárdaba

iD 0000-0001-6864-9330

Citar: Santamaría-Cárdaba, N, Franco, A, Lourenço, M y Vieira, R (2022). Educación para la Ciudadanía Global Crítica al acabar la educación obligatoria en España y Portugal: Una revisión integrativa de la literatura. REIDOCREA, 11(11), 120-134.

Área o categoría del conocimiento: Educación

Resumen: La Educación para el Desarrollo y la Ciudadanía Global (EDCG) es un objetivo cada vez más importante a nivel internacional, siendo la Agenda de Desarrollo Sostenible 2030 un ejemplo de los desafíos globales cuya solución depende de todos. Desde un cuadro referencial de EDCG Crítica, este artículo analiza críticamente la presencia de la EDCG Crítica en la legislación educativa que recoge el perfil del alumnado al acabar la escolarización obligatoria en España y Portugal, estableciendo comparaciones entre ambos países, para formular recomendaciones sobre lo que puede ser mejorado en la legislación. La metodología empleada para comprobar la presencia de la EDCG Crítica en los documentos fue una Revisión Integrativa de la Literatura. Los resultados muestran que la EDCG Crítica encuentra espacio en los documentos estudiados, pero estos presentan carencias en la utilización de conceptos fundamentales y en la identificación de ejemplos de metodologías y estrategias para impulsar una EDCG Crítica.

Palabra clave: Pensamiento crítico

Critical Global Citizenship Education at the end of compulsory education in Spain and Portugal: An integrative review of the literature

Abstract: Global Citizenship Education (GCE) is an increasingly important goal worldwide, with the 2030 Sustainable Development Agenda being one example of the global challenges whose solution depends on everyone. From a Critical GCE framework, this article critically analyzes the presence of Critical GCE in the educational legislation pertaining to students' profile at the end of compulsory education in Spain and Portugal, comparing both countries, to make recommendations on what may be improved in the legislation. The methodology used to identify the presence of Critical GCE in the documents was an Integrative Literature Review. The results show that Critical GCE is present in the documents examined, but they have gaps in fundamental concepts and in the identification of examples of methodologies, strategies, and activities to promote a Critical GCE.

Keyword: Critical thinking

\section{Introducción}

La Educación para el Desarrollo y la Ciudadanía Global (en lo sucesivo, EDCG) o para la Transformación Social es una cuestión que está adquiriendo cada vez más importancia; incluso, se han promovido iniciativas a nivel internacional que buscan apoyar e impulsar desde el sector educativo un mayor aprendizaje sobre cuestiones globales, internacionales y de desarrollo (Bourn, Hunt, Blum, y Lawson, 2016). Una de las iniciativas más relevantes es la Agenda de Desarrollo Sostenible 2030 (ONU, 2015) puesto que alcanzó un compromiso unánime por parte de todos los países integrantes de la ONU y es concebida como "un viaje compartido que debe ser asumido como propio por todos los actores, destacando que el futuro del planeta depende de todos" (Vargas y Boni, 2017: 1). 
La Agenda 2030 incluye en sus objetivos la promoción de una ciudadanía global según la cual el individuo comprenda que convive en un mundo interconectado y multicultural con problemas a una escala planetaria, y por ello se debe actuar para lograr un bienestar social (Murga-Menoyo y Novo-Villaverde, 2017). Es decir, se debe impulsar una educación global, la cual es "un proceso de crecimiento individual y colectivo que permite un cambio y una autotransformación" (Consejo de Europa, 2008: 19). La EDCG pretende formar personas comprometidas y solidarias que actúen para transformar el mundo en un lugar más humano, por lo que juega un papel indiscutible a la hora de alcanzar los objetivos marcados en esta Agenda 2030.

Adicionalmente, la sociedad cambiante actual hace necesario que las personas adquieran diversas competencias para actuar adecuadamente ante situaciones complejas y, para ello, el camino a seguir es el Pensamiento Crítico (en lo sucesivo, PC) (Franco, Almeida, y Saiz, 2014). De este modo, la EDCG y el PC se interconectan, pues la EDCG Crítica promueve una educación crítico-social que libere a la ciudadanía "de toda repetición, mecanización o dependencia del pensamiento de otros. Es una educación emancipadora que le permite a la persona transformarse y transformar su entorno de manera consciente" (Vásquez-Alape, 2012: 161-162).

Por consiguiente, la sociedad actual requiere que se formen ciudadanos globales críticos para promover una transformación hacia sociedades más humanas, lo cual conlleva la necesidad de que la legislación educativa vigente abarque este tipo de cuestiones. UNESCO (2015) ya señala la importancia de la EDCG en la educación formal, siendo normalmente trabajada "como tema transversal en el programa de estudios, como un componente integrado con diferentes temas, o como un tema independiente en el programa de estudios" (UNESCO, 2015: 48). Aquí es donde radica esta investigación, la cual pretende comprobar si la legislación educativa de España y Portugal que marca el perfil competencial que deben poseer los estudiantes al finalizar la escolarización básica (seis a 18 años) trata este tipo de cuestiones desde una perspectiva de EDCG Crítica.

\section{Revisión de la literatura}

A continuación, se explica qué es la EDCG, porqué es importante el desarrollo del PC y cómo convergen la EDCG y el PC en la EDCG Crítica.

\section{La Educación para el Desarrollo y la Ciudadanía Global}

La EDCG es una cuestión complicada de definir puesto que es un concepto muy amplio sometido a continuo cambio. Autores como Bourn (2015) y Baily, O'Flaherty, y Hogan (2017) consideran que la complejidad de definir EDCG subyace de las diversas interpretaciones que pueden afectar al concepto dependiendo del contexto social, económico o político desde el que se enfoquen. A pesar de esta dificultad, tomando como base las definiciones propuestas por Bernal y Carrica (2016), Celorio y Celorio (2011), Mesa (2014), Santamaría-Cárdaba (2020) o Santamaría-Cárdaba, Marbán, y Torrego (2019), este estudio comprende la EDCG como un proceso educativo que pretende formar ciudadanos autónomos y capaces de comprender de forma crítica los problemas que afectan a la realidad social para cambiar el mundo hacia uno más justo, humano y sostenible.

Esta concepción de la EDCG actual se ha ido viendo sometida a modificaciones a lo largo del tiempo. Concretamente, la evaluación histórica se divide en diversas generaciones o enfoques (Carrica y Bernal, 2019; Mesa, 2000). Inicialmente, entre los años 40 y 60 , emerge el primer enfoque, denominado Caritativo-asistencial, el cual se 
centraba en acciones de sensibilización sobre Educación para el Desarrollo. El segundo enfoque, llamado Desarrollista, se gestó entre los años 60-70 y buscaba transmitir las diferencias existentes entre las zonas Norte-Sur con el objetivo de buscar ayuda para los países empobrecidos. La tercera generación, Crítico-solidaria, tuvo lugar durante los años 70-80 y con ella comenzó a gestarse una visión más crítica que buscaba enseñar a la ciudadanía las causas del empobrecimiento de las zonas del Sur y mostrar la responsabilidad de los países del Norte ante esta situación de desigualdad social. Entre los años 80-90 emerge el cuarto enfoque, orientado al Desarrollo Humano y Sostenible, el cual busca lograr un desarrollo socialmente equilibrado. El quinto enfoque, que surge en los años 90, se denomina Educación para la Ciudadanía Global o Cosmopolita y busca la equidad social pretendiendo reforzar un modelo de vida social justo y democrático.

Actualmente, se está gestando la sexta generación, que autores como García-Rincón (2015) y Sainz de Murieta (2016) comprenden bajo el nombre de Educación para la Transformación Social o Educación Cosmopolita-global. Los mismos autores señalan que esta pretende formar una ciudadanía que comprenda críticamente la relación existente entre los problemas locales y globales. Sin embargo, en el presente estudio se entiende la generación emergente actualmente como EDCG Crítica, pues se centra en la perspectiva impulsada por Andreotti (2006) que remarca la necesidad de crear ciudadanos críticos para que no solo se pongan en la situación que padecen las personas que viven en los países empobrecidos, sino que actúen a favor de los Derechos Humanos tratando de impulsar una sociedad más justa. De este modo, la EDCG Crítica sobre la que se construye este estudio dispone de dos componentes esenciales ligados entre sí: la EDCG y el PC. Por esta razón, antes de profundizar el tema de la EDCG Crítica, es necesario presentar qué es el PC y cuál su importancia para la EDCG.

\section{El Pensamiento Crítico en la EDCG}

Por ser un constructo multifacético estudiado por diferentes áreas del conocimiento, el PC puede definirse de diversas maneras (Sternberg y Halpern, 2007); por ello, se presentan tres ejemplos de definiciones de áreas de conocimiento diferentes, que revelan aspectos específicos de esta competencia. Ennis, del área de Filosofía, advierte que $\mathrm{PC}$ es un pensamiento razonable y reflexivo enfocado en decidir en lo que acreditar y cómo actuar (Ennis, 2011: 1). Acorde con Saiz, del área de Psicología, el PC está orientado para la resolución eficaz, que se hace mediante "un proceso de búsqueda de conocimiento, a través de habilidades de razonamiento, de solución de problemas y de toma de decisiones, que nos permite lograr, con la mayor eficacia, los resultados deseados" (2017: 19). Por su parte, Tenreiro-Vieira y Vieira $(2000,2014)$, del área de Educación, señalan que el PC incluye un conjunto de capacidades y disposiciones, tales como: clarificar y enfocar cuestiones que sean relevantes, hacer inferencias, evaluar la credibilidad de una fuente (ejemplos de capacidades de PC); y también, apertura de espíritu, o respeto por los otros (ejemplos de disposiciones de PC). En suma, se comprende el PC como una forma superior de pensamiento que integra capacidades, disposiciones, criterios de pensamiento y conocimientos, que es útil en diversos ámbitos (sea personal, académico, profesional, o social) para "pensar bien", es decir, encontrar explicaciones, tomar decisiones y solucionar desafíos (Franco, Vieira, y Saiz, 2017).

¿Cuál es la razón de la asociación del PC con la EDCG? Ante todo, el PC es indispensable para comprender que "no pensamos tan bien como nos imaginamos, ni somos conscientes de la multitud de sesgos que padecemos, ni conocemos suficientemente bien nuestra maquinaria intelectual" (Saiz, 2017: 24). Después, y fundamentalmente, la democracia está dependiente de la competencia de los 
ciudadanos para actuar en la esfera pública. Para eso, es necesario que sepan dialogar y debatir, construir y presentar argumentos, y que actúen en conformidad (TenreiroVieira y Vieira, 2000). En este proceso, es esencial que los individuos adquieran consciencia sobre los aportes de sus padrones de pensamiento y de acción para la resolución de los problemas sociales que se intentan combatir. Con otras palabras, la EDCG debe abordar los orígenes económicos y culturales de las inequidades existentes a nivel global en la distribución de poder y riqueza, evitando la reproducción de mitos y convicciones que legitiman las desigualdades (Andreotti, 2006). Así se justifica la necesidad de una EDCG que sea Crítica, dando lugar a una combinación de EDCG con PC.

\section{La EDCG Crítica}

La educación es esencial para el desarrollo de personas críticas que impulsen una sociedad democrática y actúen contra las desigualdades y las situaciones que provocan sufrimiento a las personas (McLaren y Kincheloe, 2008; Peach y Clare, 2017). Por este motivo, los centros educativos deben potenciar el PC del alumnado para que comprendan las situaciones de injusticia y contradicción presentes en el mundo actual (Giroux, 2003). De hecho, si se pretende formar ciudadanos activos que participen en la sociedad democrática actual, es imprescindible que las personas no se limiten a comprender las cuestiones de manera superficial y que reflexionen para emitir juicios fundamentados (Naiditch, 2010; Peach y Clare, 2017). Como advierte McCowan (2009), la pedagogía crítica debe estar contemplada en los currículos educativos para que resulte más efectiva a docentes y estudiantes.

Johnson y Morris (2012) destacan los cuatro objetivos de la EDCG Crítica propuestos por Cogan, Morris, y Print (2002), y que representan las dimensiones en las que es necesario actuar: "los conocimientos, capacidades, valores y disposiciones de los ciudadanos" (Cogan et al., 2002: 4). Asimismo, Oxfam (2015) señala tres elementos clave para la EDCG: conocimientos y comprensión, capacidades, y aúnan, valores y actitudes. Acorde con los autores anteriores, los conocimientos hacen referencia a los saberes adquiridos, por ejemplo, conocer la cultura, la historia o las relaciones de poder; las capacidades relativas al PC promueven la participación activa; los valores están relacionados con el compromiso ante las desigualdades; $y$, por último, las disposiciones hacen referencia a la responsabilidad de las acciones, decisiones y el cuestionamiento activo. Sin embargo, hay cuadros conceptuales de PC (Tenreiro-Vieira y Vieira, 2014; Vieira, 2018) que señalan cuatro dimensiones del PC: las capacidades, las disposiciones, los criterios o normas de pensamiento y los conocimientos, puesto que las disposiciones y los valores están unificados. Este estudio se basa en esta premisa por lo que integra en los resultados presentados las disposiciones y los valores.

En este sentido, la EDCG Crítica actual requiere que los ciudadanos conozcan tanto el porqué de las desigualdades sociales como sus derechos, y que sean conscientes de la capacidad que poseen para actuar sobre la realidad (Mata, Ballesteros, y Padilla, 2013). Andreotti (2006) realiza una diferenciación entre EDCG "suave", la cual busca educar ciudadanos activos de acuerdo con lo que se ha establecido para ellos como un mundo idóneo, y la EDCG Crítica, cuyo objetivo es formar personas que reflexionan de forma crítica para que imaginen futuros distintos asumiendo la responsabilidad de sus actos.

La ciudadanía crítica comprendida en este artículo es similar al concepto de ciudadanía global crítica utilizado por Oxley y Morris (2013) para hacer referencia a las personas que se centran en reducir las desigualdades y defender activamente la justicia social. Por tanto, la EDCG actual ya no busca solo formar ciudadanos globales conscientes de 
su papel en el mundo bien como de los desafíos globales, sino que quiere que comprendan críticamente las causas de esta situación, reflexionen, sean responsables de sus actos y actúen para tratar de construir un mundo más justo y humano.

\section{Objetivos}

La pregunta de investigación planteada en este estudio es ¿Cuál es el espacio de la EDCG Crítica en dos documentos educativos orientadores de la educación en España y Portugal? Partiendo de esta pregunta, se han propuesto los siguientes objetivos: (i) analizar críticamente la presencia de la EDCG Crítica en la legislación educativa que recoge el perfil del alumnado al acabar la escolarización básica en España (Orden ECG/65/2015; Ministerio de Educación, Cultura y Deporte, 2015) y Portugal (Perfil dos Alunos à Saída da Escolaridade Obrigatória; Ministério da Educação, 2017), estableciendo comparaciones entre ambos países; y (ii) formular recomendaciones para solventar eventuales carencias observadas en la legislación. Se decidió analizar estos documentos porque recogen las competencias que debe poseer el alumnado al finalizar su escolarización obligatoria en ambos países, es decir, de los seis a los 18 años. Concretamente, Portugal fija la edad de escolarización obligatoria hasta los 18 años, mientras que España en los 16 años, aunque el documento analizado abarca la formación hasta los 18 años en ambos países

\section{Metodología}

La metodología empleada en este estudio consiste en la realización de una Revisión Integrativa de la Literatura (RIL). La RIL permite una síntesis, organización y discusión sobre un tema, que crean, a su vez, una mayor comprensión sobre el mismo (Botelho, Cunha, y Macedo, 2011). Esta técnica proporciona una mayor profundización y permite resumir los contenidos eficazmente, para lograr una mejora en la práctica profesional y las políticas existentes (Azevedo y Nunes, 2018). En última instancia, contribuye al desarrollo de la teoría en un área, además de ser considerada beneficiosa para la práctica y las políticas (Whittemore y Knafl, 2005).

Concretamente, este estudio realiza una RIL para comprobar la presencia de la EDCG Crítica en dos documentos legislativos orientadores de la educación en dos contextos europeos diferentes pero próximos, España y Portugal, para aportar con un análisis crítico sobre esa presencia en cada país y formular recomendaciones. Los procedimientos adoptados siguieron las orientaciones propuestas por Botelho et al. (2011) y Whittemore y Knafl (2005), y son: (1) selección de los documentos a analizar; (2) formulación de la cuestión de investigación; (3) elaboración de una matriz de síntesis; (4) análisis de los documentos; (5) validación del proceso de análisis; (6) creación de categorías conceptuales; (7) comparación de las categorías conceptuales. A continuación, presentamos detalladamente cada etapa.

\section{Procedimientos}

\section{Selección de los documentos a analizar}

Se identificaron dos documentos legislativos orientadores de la educación en los dos países que nos interesaba comparar - España y Portugal: la Orden ECD/65/2015, en España, y el Perfil del Alumnado al Finalizar la Escolarización Obligatoria, en Portugal. A partir del análisis de estos documentos, el objetivo era detectar si la EDCG Crítica efectivamente se encuentra plasmada en dichos documentos educativos, e identificar similitudes y diferencias entre los documentos de ambos países. 


\section{Formulación de la cuestión de investigación}

Tras seleccionar los documentos que se van a analizar, se planteó la cuestión de investigación que iba a guiar el análisis de los documentos y, por extensión, de los resultados. La cuestión de investigación planteada elegida fue la misma que dirigió el presente estudio: ¿Cuál es el espacio de la EDCG Crítica en dos documentos educativos orientadores de la educación en España y Portugal?

\section{Elaboración de una matriz de síntesis}

Teniendo como base la cuestión de investigación formulada para extraer la información referente a la EDCG de los dos documentos, se utilizó una matriz de síntesis construida para ello, la cual permite extraer y organizar la información de cada documento y, después, comparar estos datos (Botelho et al., 2011). La matriz de síntesis presentaba un conjunto de criterios de análisis que son clave en la literatura sobre EDCG Crítica, y que se podían identificar en los propios documentos: "conocimientos"; "capacidades"; "disposiciones"; "metodología de enseñanza-aprendizaje"; y "finalidades educativas". A continuación, la Tabla 1 muestra las categorías de análisis empleadas, las cuales parten de la revisión de la literatura sobre EDCG realizada en líneas anteriores.

Tabla 1. Categorías de análisis.

\begin{tabular}{|c|c|}
\hline CATEGORIAS & SUBCATEGORIAS \\
\hline Dimensiones de la EDCG Crítica & $\begin{array}{l}\text { Conocimientos } \\
\text { Capacidades } \\
\text { Disposiciones }\end{array}$ \\
\hline $\begin{array}{l}\text { Metodología de enseñanza-aprendizaje } \\
\text { Finalidades educativas }\end{array}$ & \\
\hline
\end{tabular}

\section{Análisis de los documentos}

El documento legislativo de la educación en España comenzó siendo analizado por una de las autoras de este artículo, investigadora en España y nativa de ese país. Por otro lado, el documento legislativo de la educación en Portugal empezó a ser analizado por otra de las autoras, investigadora en Portugal y nativa de ese país. El análisis de cada documento fue realizado a partir de la matriz de síntesis construida anteriormente. A medida que el documento de cada país era examinado, cada investigadora completaba su matriz de síntesis con la información relevante en cada uno de los cinco criterios de análisis establecidos. Para hacer este registro de la información, la unidad de análisis fue la frase (terminada en punto final o punto y coma). Así, en las matrices de síntesis, se rellenó cada tabla con extractos extraídos de los documentos analizados.

\section{Validación del proceso de análisis}

Tras este análisis inicial, cada investigadora analizó la matriz de síntesis de su compañera para validar su proceso de análisis. En esta etapa, se tomaron decisiones sobre la precisión de los criterios de análisis construidos anteriormente y se hicieron los ajustes necesarios.

Seguidamente, dos investigadores fluentes en las dos lenguas, español y portugués, y también autores de este artículo, analizaron, separadamente y en conjunto, en momentos distintos, las dos matrices de síntesis para mejorar el proceso de validación del análisis. En base a las aportaciones de los cuatro investigadores fue necesario tomar nuevas decisiones sobre la precisión de los criterios de análisis construidos y hacer modificaciones. Por ejemplo, decidir incluir disposiciones y valores en la misma categoría, aunque en los documentos, a veces aparezcan separadamente. 


\section{Creación de categorías conceptuales}

Con las dos matrices de síntesis completas y validadas por los cuatro investigadores, la siguiente etapa fue crear categorías más sencillas con la información contenida en los extractos de cada matriz de síntesis. Por ejemplo, si en la matriz de síntesis del documento portugués, para el criterio de análisis "disposiciones", se había identificado el extracto "livre, autónomo, responsável e consciente de si próprio e do mundo que o rodeia;" (Ministério da Educação, 2017: 15), fueron creadas categorías más sencillas para representar el contenido de ese extracto, como: autonomía; responsabilidad; concienciación. Este proceso fue realizado con todos los extractos. Al final de este proceso existían categorías que se repetían, por eso, al final, resultó, para cada criterio de análisis, un grupo de categorías conceptuales comunes. Por ejemplo, para el criterio de análisis "disposiciones" en la matriz de síntesis del documento portugués, se encontraron categorías conceptuales como: responsabilidad; autonomía; acción ética; solidaridad.

\section{Comparación de las categorías conceptuales}

Para terminar el proceso de análisis fue construida una tabla única con categorías conceptuales para cada criterio de análisis definido -en lo sucesivo llamado dimensióny se fue comparando la información recogida en los dos documentos analizados, para poder dar respuesta a la pregunta de investigación.

\section{Resultados}

\section{Dimensiones de la EDCG Crítica}

Los criterios de análisis propuestos en la matriz se corresponden con las dimensiones de la EDCG y del PC: conocimientos, capacidades y disposiciones (esta última, como se explicó anteriormente, integra los valores).

1.1. Conocimientos. Los datos obtenidos en la dimensión de conocimientos sobre EDCG al analizar la legislación citada de España y Portugal se han resumido en la Tabla 2. Las semejanzas se han remarcado con negrita.

Tabla 2. Comparación de los conocimientos encontrados en los documentos de España y Portugal CONOCIMIENTOS

\begin{tabular}{|c|c|}
\hline ESPAÑA & PORTUGAL \\
\hline $\begin{array}{l}\text { Saber cómo las tecnologías pueden permitir diferentes formas de } \\
\text { participación para crear contenidos que produzcan un beneficio } \\
\text { común. }\end{array}$ & $\begin{array}{l}\text { Reconhecer a importância das diferentes áreas para a } \\
\text { sustentabilidade social, cultural, económica e ambiental de } \\
\text { Portugal e do mundo. }\end{array}$ \\
\hline $\begin{array}{l}\text { Conocer conceptos sobre la organización del trabajo, la } \\
\text { igualdad, no discriminación entre hombres y mujeres, y entre } \\
\text { diferentes grupos étnicos o culturales, la sociedad y la } \\
\text { cultura. }\end{array}$ & $\begin{array}{l}\text { Conhecer e respeitar e agir de acordo com os princípios e } \\
\text { direitos fundamentais da sociedade democrática: respeitar } \\
\text { a dignidade humana; exercer uma cidadania plena; ser } \\
\text { solidário com os outros; respeitar a diversidade cultural; }\end{array}$ \\
\hline $\begin{array}{l}\text { Comprender las dimensiones socioeconómica e intercultural de } \\
\text { las sociedades europeas. }\end{array}$ & $\begin{array}{l}\text { respeitar o debate democrático; negociar a resolução de } \\
\text { conflitos; trabalhar colaborativamente para o bem comum. }\end{array}$ \\
\hline
\end{tabular}

Conocer la realidad social del mundo en el que se vive, sus conflictos y las motivaciones de estos.

1.2. Capacidades. La revisión de los documentos analizados en España y Portugal da lugar a una síntesis de las características de la dimensión de la EDCG Crítica relativa a las 
capacidades, la cual se encuentra en la Tabla 3. Las semejanzas se han remarcado con negrita.

Tabla 3. Comparación de las capacidades identificadas en los documentos de España y Portugal CAPACIDADES

\begin{tabular}{ll}
\hline ESPAÑA & PORTUGAL \\
\hline Aprendizaje a lo largo de la vida. & Tomar decisões livres e fundamentadas. \\
Capacidad crítica en la toma de decisiones. & Analisar e questionar criticamente a realidade. \\
Actuar a favor de la sostenibilidad del bienestar social. & Selecionar e avaliar a informação. \\
Analizar, interpretar y cotejar la información. & Formular hipóteses. \\
$\begin{array}{l}\text { Conocer de forma crítica los conceptos de democracia, justicia, } \\
\text { igualdad, ciudadanía y derechos humanos y civiles. }\end{array}$ & Participar civicamente. \\
Liderazgo y responsabilidad. & Ser criativo. \\
Trabajar individualmente y en equipo. & Trabalhar colaborativamente. \\
Comunicarse de manera constructiva en diferentes entornos & Capacidade de comunicação. \\
sociales y culturales. & Saber lidar com a mudança e incerteza. \\
Comprender y analizar de manera crítica. & Valorizar a aprendizagem contínua. \\
& Pensar criticamente. \\
\hline
\end{tabular}

1.3. Disposiciones. La Tabla 4 sintetiza las características relativas a la dimensión de las disposiciones propia la EDCG Crítica en ambos países. Las semejanzas se han remarcado con negrita.

Tabla 4. Comparación de las disposiciones identificadas en los documentos de España y Portugal

\begin{tabular}{|c|c|}
\hline DISPOSICIONES & \\
\hline ESPAÑA & PORTUGAL \\
\hline $\begin{array}{l}\text { Ser responsable con la conservación de los recursos } \\
\text { naturales. }\end{array}$ & $\begin{array}{l}\text { Consciência e responsabilidade pela sustentabilidade } \\
\text { ecológica. }\end{array}$ \\
\hline Interés por el desarrollo socioeconómico y su contribución al & Espírito empreendedor. \\
\hline Dienestar social de toda la podiacion. & Autonomia. \\
\hline Interés en la comunicación intercultural. & Auto-confiança. \\
\hline Interés sobre la diversidad de valores. & Resiliência. \\
\hline $\begin{array}{l}\text { Comprometerse personal y colectivamente con la mejora de } \\
\text { la realidad social. }\end{array}$ & Espírito de iniciativa. \\
\hline Participar de forma activa y eficaz en la vida social y & Concretizar projetos com responsabilidade e autonomia. \\
\hline profesional. & Integrar-se ativamente na sociedade. \\
\hline Actuar de forma ética. & Responsabilidade. \\
\hline Responsabilidad. & Liberdade. \\
\hline Tolerancia. & Integridade. \\
\hline Empatía. & Respeito por si/outros. \\
\hline Actuar para favorecer la convivencia social. & Ação ética. \\
\hline Respetar las diferencias. & Solidariedade. \\
\hline Realización de juicios críticos. & Rigor. \\
\hline Rigor. & Perseverança. \\
\hline Autonomía. & Reflexividade crítica. \\
\hline & Conscienciosidade. \\
\hline
\end{tabular}




\section{La EDCG Crítica en la metodología de enseñanza-aprendizaje}

La Tabla 5 presenta la metodología de enseñanza-aprendizaje marcada en la legislación analizada en España y Portugal y remarca las semejanzas en negrita.

Tabla 5. Comparación de la metodología de enseñanza-aprendizaje identificada en los documentos de España y Portugal

\begin{tabular}{|c|c|}
\hline \multicolumn{2}{|l|}{ METODOLOGÍA DE ENSEÑANZA-APRENDIZAJE } \\
\hline ESPAÑA & PORTUGAL \\
\hline Docente como orientador, promotor y facilitador del desarrollo & Aprendizagem baseada na cooperação e colaboração. \\
\hline competencial en el alumn & Ação educativa coerente e flexível. \\
\hline Aprendizaje basado en problemas. & Papel autónomo e ativo do aluno. \\
\hline $\begin{array}{l}\text { Despertar y mantener la motivación hacia el aprendizaje en el } \\
\text { alumnado. }\end{array}$ & Trabalho de projeto. \\
\hline Papel del alumno, activo y autónomo, consciente de ser el & Discussão de pequeno grupo e debate. \\
\hline responsable de su aprendizaje. & Exploração de recursos. \\
\hline Metodologías activas y contextualizadas. & Pesquisa. \\
\hline $\begin{array}{l}\text { Aprendizaje en equipo de forma cooperativa y } \\
\text { colaborativa. }\end{array}$ & Aprendizagem orientada à ação. \\
\hline Trabajo por proyectos. & \\
\hline Aprendizaje orientado a la acción. & \\
\hline
\end{tabular}

\section{La EDCG Crítica en las finalidades educativas}

Como se muestra en la Tabla 6, las finalidades educativas de ambos países poseen semejanzas, las cuales se han remarcado en negrita, y señalan la necesidad de formar ciudadanos críticos y desarrollar el PC.

Tabla 6. Comparación de las finalidades educativas identificadas en los de España y Portugal

\begin{tabular}{|c|c|}
\hline INALIDADES EDUCATIVAS & \\
\hline ESPAÑA & PORTUGAL \\
\hline Lograr que los individuos alcancen un pleno desarrollo & Desencadear a aprendizagem e a qualificação individual. \\
\hline $\begin{array}{l}\text { personal, social y profesional que se ajuste a las } \\
\text { demandas de un mundo globalizado. }\end{array}$ & $\begin{array}{l}\text { Contribuir para o desenvolvimento de competências para } \\
\text { o exercício de uma cidadania ativa e criativa, para }\end{array}$ \\
\hline Educar ciudadanos activos. & responder aos desafios globais e à imprevisibilidade. \\
\hline Promover el aprendizaje a lo largo de la vida. & Contribuir para o desenvolvimento de competências para \\
\hline Fomentar el respeto por las normas de convivencia. & $\begin{array}{l}\text { intervir no quadro da construção de uma sociedade mais justa } \\
\text { e democrática. }\end{array}$ \\
\hline Estimular el respeto por los Derechos Humanos & Formar para uma consciência de sustentabilidade. \\
\hline $\begin{array}{l}\text { Llegar a entender el diálogo como herramienta para la } \\
\text { resolución de conflictos. }\end{array}$ & Promover a formação contínua. \\
\hline Despertar una actitud de curiosidad hacia el aprendizaje. & $\begin{array}{l}\text { Fomentar a cultura científica que permite compreender, tomar } \\
\text { decisões e intervir sobre as realidades naturais e sociais no }\end{array}$ \\
\hline Promover las acciones orientadas a la conser & mundo. \\
\hline & explorar temas diferencia \\
\hline Desarrollar el pensamiento científico. & para \\
\hline $\begin{array}{l}\text { Lograr la adquisición de conocimientos, contrastar ideas y } \\
\text { aplicar los descubrimientos al bienestar social. }\end{array}$ & $\begin{array}{l}\text { Fomentar a adaptação a novos contextos e novas estruturas, } \\
\text { e a novas funções. }\end{array}$ \\
\hline
\end{tabular}




\section{Discusión}

El presente artículo tenía como objetivo inicial analizar la presencia de la EDCG Crítica en la legislación que recoge el perfil del alumnado al acabar la escolarización básica en España (Orden ECG/65/2015) y Portugal (Perfil dos Alunos à Saída da Escolaridade Obrigatória). Ese análisis comparativo de la presencia de la EDCG Crítica en la legislación de ambos países fue hecho para, en segunda instancia, elaborar un análisis crítico sobre similitudes y diferencias, y formular recomendaciones sobre carencias en la legislación.

Para comprobar la presencia de la EDCG Crítica en los dos documentos, se realizó una RIL para sintetizar, organizar y comparar la información recogida y, también, discutir los datos y contribuir a la comprensión del tema en análisis (Botelho et al., 2011; Whittemore y Knafl, 2005). Los resultados fueron analizados en tres grandes temas: dimensiones de la EDCG Crítica (conocimientos, capacidades y disposiciones); la EDCG (Crítica) en la metodología de enseñanza-aprendizaje; y la EDCG (Crítica) en las finalidades educativas. En cada uno de estos tres temas, se observó que tanto había categorías conceptuales comunes como distintas en la legislación educativa de España y Portugal.

\section{El espacio de la EDCG Crítica en las dimensiones}

En lo que concierne a las dimensiones de la EDCG Crítica se verificó, con respecto a los conocimientos que deberán ser el objetivo que orienta la escolarización, que la legislación de ambos países pretende fomentar el bien común. Por una parte, en la legislación española se destaca la necesidad de aplicar las tecnologías para lograr un beneficio común, de conocer conceptos relacionados con la equidad de género, y de comprender la realidad socioeconómica y la diversidad cultural. Por otra parte, en la legislación portuguesa se busca conocer nociones sobre sustentabilidad socioeconómica, cultural y ambiental, y comprender los derechos de las sociedades democráticas para respetar las diferentes culturas o trabajar colaborativamente para el bien común. Así, no hay diferencias significativas entre ambos países sobre los conocimientos que el alumnado debe desarrollar durante su escolaridad obligatoria.

Pero ¿será que el espacio de la EDCG Crítica en los documentos educativos en España y Portugal representa bien los conocimientos enfatizados por la literatura? Según diversos autores (Cogan et al., 2002; Johnson y Morris, 2012; Oxfam, 2015), los conocimientos sobre EDCG deben tratar las relaciones y la distribución de poder presentes en la cultura e historia, buscando que los ciudadanos conozcan las razones socioeconómicas que crean y mantienen las desigualdades sociales, para que actúen a favor de la justicia social (Andreotti, 2006; McLaren y Kincheloe, 2008; Peach y Clare, 2017; Oxley y Morris, 2013). De hecho, Andreotti (2006) refuerza que la EDCG Crítica presupone el razonamiento crítico aliado a la actuación.

La legislación de España parece instar a estos aspectos cuando se refiere, por ejemplo, a conocer "la realidad social del mundo en el que se vive, sus conflictos y las motivaciones de los mismos" (Ministerio de Educación, Cultura y Deporte, 2015: 6999). Sin embargo, quizás sea necesario reforzar (en el caso de España) o crear (en el caso de Portugal) una recomendación referente a la construcción de conocimiento sobre el concepto de injusticia social, la cual deriva en inequidades que toda la ciudadanía debe ayudar a corregir a través de su reflexión crítica y acción. Por tanto, este es un principio esencial en la EDCG Crítica que necesita ser reforzado en las legislaciones.

Respecto a las capacidades, la legislación de ambos países señala que a lo largo de la escolaridad obligatoria se deben desarrollar capacidades como: pensar críticamente 
para tomar decisiones razonables y actuar de forma cívica, saber trabajar colaborativamente, o bien aprender continuamente. Por una parte, en la legislación española se destaca la capacidad de liderazgo, la responsabilidad, la importancia de actuar a favor del bienestar social. Por otra parte, en la legislación portuguesa se resalta la capacidad de comunicación y de lidiar con la incertidumbre y los cambios. Así, a pesar de las capacidades destacadas en cada legislación, todas ellas tienen bien presentes las capacidades de PC identificadas en la literatura (Franco et al., 2017; Saiz, 2017; Tenreiro-Vieira y Vieira, 2000), tal como "analizar, interpretar y cotejar la información" (en España) o "formular hipótesis" y "tomar decisões livres e fundamentadas" (en Portugal). De este modo, la respuesta a la pregunta ¿el espacio de la EDCG Crítica en los documentos educativos en España y Portugal representa bien las capacidades enfatizadas por la literatura? es afirmativa.

En cuanto a las disposiciones, la legislación de ambos países pretende que, al finalizar la escolaridad obligatoria, el alumnado haya desarrollado la curiosidad, la concienciación, el rigor, el respeto, la responsabilidad para actuar activamente y de forma ética en la sociedad. Por una parte, en la legislación española se destaca la tolerancia, la disciplina, la paciencia, la empatía, el actuar favoreciendo la convivencia en sociedad y el estar comprometido y dispuesto a superar prejuicios. Por otra parte, en la legislación portuguesa se resalta la integridad, la libertad, la autonomía, la solidaridad y la perseverancia. Así como en el caso de las capacidades, a pesar de las disposiciones destacadas en cada legislación, ellas se refieren a las disposiciones de PC identificadas en la literatura (Franco et al., 2017; Saiz, 2017; Tenreiro-Vieira y Vieira, 2000) y que son valoradas en la EDCG Crítica, tal como "respectar las diferencias", "empatía", "comprometerse personal y colectivamente con la mejora de la realidad social".

Una vez más, el espacio de la EDCG Crítica en los documentos educativos en España y Portugal parece representar bien las disposiciones enfatizados por la literatura. Sin embargo, cabe señalar que parece haber alguna confusión en la legislación de ambos países sobre lo que específicamente es capacidad y lo que es disposición. Al realizar el análisis en la matriz de síntesis fue necesario reorganizar conceptos, pues frecuentemente resultaba alguna sobreposición entre las dos - capacidades y disposiciones -, con conceptos identificados como capacidades pero que eran, de hecho, disposiciones y viceversa. Consecuentemente, se sugiere que las legislaciones sean analizadas por expertos que ayuden a distinguir e identificar con precisión qué es capacidad y qué es disposición.

\section{El espacio de la EDCG Crítica en la metodología de enseñanza-aprendizaje}

En relación con la EDCG Crítica en la metodología de enseñanza-aprendizaje, se apreciaron diferencias significativas entre ambos países. En la legislación española se destaca una metodología de enseñanza-aprendizaje activa y contextualizada, orientada a la acción, basada en el trabajo por proyectos y en la resolución de problemas, que emplee como estrategias evaluativas la autoevaluación, la evaluación entre iguales y la coevaluación. En la legislación portuguesa se resalta una acción educativa flexible y coherente, que fomente la investigación, el debate, la exploración de recursos y la discusión en pequeños grupos. Sin embargo, aunque compartan metodologías similares, la legislación española parece profundizar más sobre la metodología de enseñanza-aprendizaje que deber ser utilizada durante la escolaridad obligatoria, ofreciendo más ejemplos específicos que la legislación portuguesa. Asimismo, la legislación española contempla los ámbitos de la educación formal, no formal e informal, mientras que en Portugal no se resalta explícitamente el contexto informal (aunque curiosamente indique la familia, por ejemplo, como un actor interviniente en el proceso de enseñanza-aprendizaje). Respecto a los actores que intervienen en este proceso, la 
legislación española se refiere al profesorado y al alumnado, y la legislación portuguesa destaca el papel del profesorado, de las familias, de los encargados de educación, de los gestores y líderes políticos.

Por tanto, ¿el espacio de la EDCG Crítica en los documentos educativos en España y Portugal representa bien la metodología de enseñanza-aprendizaje enfatizada por la literatura? Según Castro (2013) o Aguado (2011), la metodología de enseñanzaaprendizaje de la ED debe ser activa, participativa, contextualizada en un entorno que desarrolle el PC, favorecer el aprendizaje autónomo, desarrollar el pensamiento global y tratar la EDCG desde un enfoque holístico. Además, sería importante que tales documentos instruyesen sobre cómo trabajar los mismos temas de la EDCG en áreas y asignaturas distintas del currículo (Boqué, Pañellas, Alguacil, y García, 2014; Figueiredo, 2018). Consecuentemente, podrá recomendarse que la legislación de ambos países profundice aspectos relativos a la metodología de enseñanzaaprendizaje. Asimismo, la legislación española puede considerar otros actores que intervienen en el proceso de enseñanza-aprendizaje además del profesorado y del alumnado, mientras que la legislación portuguesa puede profundizar más la metodología de enseñanza-aprendizaje que deberá ser utilizada al largo de la escolaridad obligatoria, ofreciendo más ejemplos específicos de esa metodología. Además, puede ser importante explicitar el ámbito de la educación informal en la legislación, ya que, implícitamente, se valora ese ámbito.

\section{El espacio de la EDCG Crítica en las finalidades educativas}

La presencia de la EDCG Crítica en las finalidades educativas de ambas legislaciones destaca la formación de una ciudadanía crítica, el desarrollo del pensamiento científico del estudiantado, la capacidad para responder a los desafíos del mundo globalizado y el reto del aprendizaje a lo largo de la vida. En la legislación española se destaca el respeto por las normas de convivencia y por los Derechos Humanos, el desarrollo del espíritu crítico, el fomento del diálogo para la resolución de conflictos, la curiosidad ante el aprendizaje, la conservación y protección del medio natural, la adquisición de conocimientos y su aplicación para lograr el bienestar social. En la legislación portuguesa se resalta la adquisición de competencias para construir una sociedad más justa y democrática, el desarrollo de una conciencia de sustentabilidad, la capacidad de intervenir sobre la realidad natural y social del mundo, la adaptación a los nuevos contextos y funciones. Por tanto, no hay diferencias significativas entre ambas legislaciones sobre las finalidades educativas que deben guiar la formación del alumnado durante la escolaridad obligatoria.

Pero ¿el espacio de la EDCG Crítica en los documentos educativos en España y Portugal representa las finalidades educativas enfatizadas por la literatura? Esta es una cuestión importante puesto que la competencia global aparece por la primera vez como un aspecto a evaluarse en el alumnado en el test PISA (OCDE, 2018, p. 7). Según Ortega (2007), los objetivos de la EDCG se resumen en sensibilizar, formar, concienciar y movilizar. Pérez-Pérez (2016) resalta como finalidades "trabajar por la justicia y el cambio social en este mundo globalizado en el que vivimos" (p. 212). Bourn, McKenzie, y Shiel (2006) y el Consejo de Europa (2008) también comentan diversos objetivos de la EDCG, como: formar personas conscientes de sus responsabilidades siendo ciudadanos activos a favor de la justicia social y la sostenibilidad. Por consiguiente, comparando las finalidades educativas vehiculadas en las legislaciones con lo que sugiere la literatura, el espacio de la EDCG Crítica en los documentos educativos en España y Portugal parece estar representado. 


\section{Conclusiones}

Los objetivos que se pretendían concretizar con en el presente trabajo se han cumplido: (i) analizar críticamente la presencia de la EDCG Crítica en la legislación educativa que recoge el perfil del alumnado al acabar la escolarización básica en España y Portugal, estableciendo comparaciones entre ambos países; y (ii) formular recomendaciones para mejorar eventuales carencias en la legislación. Tal aporte podrá ser relevante para ayudar a concretizar la EDCG Crítica en la teoría y, especialmente, en la práctica educativa de ambos países.

Importa considerar que, según un estudio comparativo de las políticas de implementación de la EDCG en la enseñanza básica de 10 países europeos, incluyendo España y Portugal, se concluyó que la EDCG aún no está plenamente incorporada en los sistemas educativos de ninguno de ellos, a pesar de la abertura que existe para que tal venga a ocurrir y que es visible, por ejemplo, con la existencia de documentos de legislación educativa, como los analizados en el presente artículo, que buscan dar un encuadramiento legal y fomentar su concretización (Inguaggiato y Coelho, 2017).

En relación con los conocimientos que el alumnado deberá construir durante su escolaridad obligatoria, parece ser necesario reforzar y crear en ambos países la construcción de conocimiento sobre los orígenes y los factores que suportan la manutención de las diversas formas de injusticia social. Respecto a las capacidades y las disposiciones que el alumnado deberá desarrollar durante su escolarización, parece ser necesaria una mejor precisión en la utilización de los conceptos en la legislación de ambos países, para evitar que haya sobreposición y confusión.

Asimismo, la metodología de enseñanza-aprendizaje empleada durante la escolaridad obligatoria del alumnado muestra que la legislación española necesita considerar otros actores que intervienen en el proceso de enseñanza-aprendizaje además del profesorado y del alumnado; además, la legislación portuguesa debe profundizar más, ofrecer ejemplos específicos sobre la metodología que se debe utilizar, y hacer referencia explícitamente al ámbito informal. En los dos casos, es necesario que la formación (inicial y continuada) de profesores y formadores de profesores ayude el profesorado a repensar sus prácticas e identidad profesional de modo a concretizar la EDCG en la escuela (Andrade y Lourenço, 2019; Lourenço, 2018a, 2018b).

Cabe destacar que este análisis posee ciertas limitaciones, pues analiza los documentos que establecen el perfil del alumnado al acabar la escolarización obligatoria pero no estudia de forma precisa la normativa establecida para cada uno de los niveles, la cual podrá aportar datos aún más detallados y es una línea futura que se debe tener en cuenta para profundizar en esta cuestión. Como líneas de futuro, podrá ser un aporte interesante llevar a cabo entrevistas a actores educativos y a políticos, para complementar los datos aquí presentados.

Finalmente, volviendo a la pregunta inicial, se puede señalar que los resultados del análisis realizado vislumbran que la EDCG Crítica parece encontrar espacio en los documentos educativos en España y Portugal estudiados. Sin embargo, parece ser necesario seguir trabajando para aumentar ese espacio, a través de la utilización de un lenguaje más claro, preciso y coherente en la legislación, y de la aplicación del saber emergente de la literatura en EDCG Crítica para la reformulación de las legislaciones educativas. Además, es necesario compartir ejemplos claros de metodologías, estrategias y tareas que permitan promover una EDCG que va más allá de una simple sensibilización para el otro y el mundo (EDCG "suave"), para una EDCG Crítica y transformadora. Esto es fundamental para que en los centros educativos se pueda 
concretizar lo que está preconizado en la legislación de los dos países. Incluso, resulta necesario evaluar sí los discursos de EDCG Crítica se concretizan en la práctica y de qué modo, pues el profesorado puede considerar que aún no posee la formación necesaria para incluir la EDCG Crítica en sus prácticas pedagógicas cotidianas. Así, se sugiere escuchar estos profesionales y otros actores educativos, a través de entrevistas, para acceder no solo a sus representaciones y convicciones, sino también a sus prácticas, reportadas de forma a poder identificar necesidades de formación.

Por consiguiente, este estudio es una aportación relevante, en particular para la mejoría de las políticas educativas que intentan asegurar la concretización de la EDCG Crítica en la práctica. Esto es así porque, si se toman como base los resultados obtenidos, se aprecia la necesidad de esforzarse por solventar las carencias presentes en estas normativas y formar una ciudadanía global crítica que transforme el mundo en un lugar mejor.

\section{Referencias}

Aguado, G (2011). Educación para el desarrollo y la ciudadanía global. Madrid: INTERED.

Andrade, Al, \& Lourenço, M (2019). Educação para a cidadania global e identidade profissional: Um estudo de caso na formação inicial de professores. En Nuno Fraga (coord.), O professor do século XXI em perspetiva comparada: Transformações e desafios para a construção de sociedades sustentáveis (pp. 537-555). Funchal: Universidade de Madeira.

Andreotti, V (2006). Soft versus critical global citizenship education. Policy \& Practise, $3(1), 40-51$

Azevedo, M, \& Nunes, D (2018). Que sugerem as pesquisas sobre os métodos de ensino para alunos com transtorno do espectro autista? Uma revisão integrativa da literatura. Arquivos Analíticos de Politicas Educativas, 24(26), 1-24.

Baily, F, O'Flaherty, J, \& Hogan, D (2017). Exploring the nature and implications of student teacher engagement with development education initiatives. Irish Educational Studies, 36(2), 185-201.

Bernal, A y Carrica, S (2016). Educación para el desarrollo y enseñanza obligatoria. Revista Española de Pedagogía, 265, 499515.

Boqué, M, Pañellas, M, Alguacil, M y García, L (2014). La cultura de paz en la educación para la ciudadanía y los derechos humanos en los libros de texto de educación primaria. Perfiles Educativos, $36(146), 80-97$.

Botelho, L, Cunha, C, \& Macedo, M (2011). Método da revisão integrativa nos estudos organizacionais. Revista Eletrônica Gestão e Sociedade, 4(11), 121-136.

Bourn, D (2015). The theory and practice of development education: A pedagogy for global social justice. New York: Routledge.

Bourn, D, Hunt, F, Blum, N, \& Lawson, H (2016). Primary education for global learning and sustainability. York: Cambridge Primary Review.

Bourn, D, McKenzie, A, \& Shiel, C (2006). The global university: The role of the curriculum. London: DEA.
Carrica, S \& \& Bernal, A (2019). The current framework of Development Education in Spain: achievements and challenges. Iberoamerican Journal of Development Studies, 8, 98-127.

Castro, M (2013), Dónde y cómo introducir la educación para el desarrollo en los centros educativos. Madrid: CCS

Celorio, JJ y Celorio, G (2011). ¿Educación? para el ¿desarrollo? Pueblos. Revista de Información y Debate, 46, 1-3.

Cogan, J, Morris, P, \& Print, M (2002). Civic education in the AsiaPacific region: Case studies across six societies. New York: Routledge.

Consejo de Europa. (2008). Pautas para una educación global conceptos y metodología sobre educación global para educadores y responsables de políticas en materia educativa. Lisboa: Centro Norte-Sur del Consejo de Europa.

Ennis, R (2011). The nature of critical thinking: An outline of critical thinking dispositions and abilities. https://education.illinois.edu/docs/default-source/facultydocuments/robertennis/thenatureofcriticalthinking_51711_000.pdf?sfvrsn=7bb51288 $-2$

Figueiredo, A (2018). A educação para o desenvolvimento e para a cidadania global no currículo de português: Relato de uma experiência numa turma de $4 .^{\circ}$ ano. Aveiro: Universidade de Aveiro.

Franco, A, Almeida, L y Saiz, C (2014). Pensamiento crítico: Reflexión sobre su lugar en la Enseñanza Superior. Educatio Siglo XXI, 2(32), 81-96.

Franco, A, Vieira, R, \& Saiz, C (2017). O pensamento crítico: As mudanças necessárias no contexto universitário. Revista de Estudios e Investigación en Psicología y Educación, volumen extraordinario, 7, 12-16.

García-Rincón, C (2015), "Siete claves para la consultoría de EPDH en centros educativos desde un enfoque de aprendizaje global, competencial y experiencial", Revista Internacional sobre Investigación en Educación Global y para el Desarrollo, 8, 5-37.

Giroux, H (2003). "Pedagogy of the depressed: Beyond the new politics of cynicism", in M Peters, C Lankshear, \& M Olssen (Eds.), 
Critical theory and the human condition: Founders and praxis, New York, Peter Lang (143-168)

Inguaggiato, C, \& Coelho, LS (2017). Políticas de implementação da educação para a cidadania global no ensino básico: Análise comparativa realizada no âmbito do projeto Global Schools. Sinergias, 5, 55-73.

Johnson, L, \& Morris, P (2012). Critical citizenship education in England and France: A comparative analysis. Comparative Education, 3(48), 283-301.

Lourenço, M (2018a). Cidadania global e integração curricular: Desafios e oportunidades nas vozes de formadores de professores. Indagatio Didactica, 10(2), 9-27.

Lourenç, M (2018b). Internationalizing teacher education curricula: Opportunities for academic staff development. On the Horizon, 2(26), 157-169.

Mata, P, Ballesteros, B y Padilla, M (2013). Ciudadanía participativa y transformadora: Análisis de discursos y propuestas de aprendizaje. Teoría de la Educación, 25, 49-68.

McCowan, T (2009). Rethinking citizenship education: A curriculum for participatory democracy. London: Continuum.

McLaren, P (2008). Pedagogía crítica: De qué hablamos, dónde estamos. Barcelona: Graó.

Mesa, M (2000). La educación para el desarrollo: Entre la caridad y la ciudadanía global. Papeles de Cuestiones Internacionales, 70 , 11-26.

Mesa, M (2014). Precedentes y evolución de la educación para el desarrollo: Un modelo de cinco generaciones. Sinergias, 1, 24-56.

Murga-Menoyo, MA y Novo-Villaverde, M (2017). Sostenibilidad, desarrollo «glocal» y ciudadanía planetaria: Referentes de una pedagogía para el desarrollo sostenible. Teoría de la Educación, 1(29), 55-78.

Naiditch, $F(2010)$. Critical pedagogy and the teaching of reading for social action. Critical Questions in Education, 1(2), 94-107.

Ortega, M (2007), Estrategia de educación para el desarrollo de la cooperación española. Madrid, Ministerio de Asuntos Exteriores y de Cooperación.

OCDE (2018). Preparing our youth for an inclusive and sustainable world - The OECD PISA global competence framework. Francia: OCDE.

ONU (2015). Transformar nuestro mundo: La Agenda 2030 para el desarrollo sostenible, en: https://unctad.org/meetings/es/SessionalDocuments/ares70d1_es. pdf

Oxfam (2015). Global citizenship in the classroom - A guide for teachers. Oxford: Oxfam.

Oxley, L, \& Morris, P (2013). Global citizenship: A typology for distinguishing its multiple conceptions. British Journal of Educational Studies, 3(61), 301-325.

Peach, S, \& Clare, R (2017). Global citizenship and critical thinking in higher education curricula and police education. Journal of Pedagogic Development, 2(7), 46-57.
Pérez-Pérez, I (2016). Education for development: The key to understanding. Revista Educación y Desarrollo Social, 2(10), 196215.

Saiz, C.(2017). Pensamiento crítico y cambio. Madrid: Ediciones Pirámide.

Sainz de Murieta, J (2016). El papel de las enseñanzas técnicas universitarias en la cooperación universitaria al desarrollo y en la formación de ciudadanía global y transformadora. IKASTORRATZA, e-Revista de Didáctica, 17, 1-7.

Santamaría-Cárdaba, N (2020). Buscando la salida del laberinto: análisis de la definición de educación para el desarrollo. Educação e Pesquisa, 46, 1-17

Santamaría-Cárdaba, N, Marbán, JM y Torrego, L (2019). Diagnóstico de la Educación para el Desarrollo en áreas rurales: un análisis correlacional de las actitudes de la población europea. Revista de Fomento Social, 294, 177-200.

Sternberg, R, \& Halpern, D (2007). Critical thinking in psychology. New York: Cambridge University Press.

Tenreiro-Vieira, C y Vieira, R (2000). Promover o pensamento crítico dos alunos: Propostas concretas para a sala de aula. Porto: Porto Editora.

Tenreiro-Vieira, C y Vieira, R (2014). Construindo práticas didáticopedagógicas promotoras da literacia científica e do pensamento crítico. Madrid: Iberciencia.

UNESCO (2015). Educación para la ciudadanía mundial: Temas y objetivos de aprendizaje. Paris: UNESCO.

Vargas, M y Boni, A (2017). La investigación colectiva para la transformación social hacia la ciudadanía global y el desarrollo sostenible. Revista Española de Desarrollo y Cooperación, 41, 4153.

Vásquez-Alape, L (2012). Actitud y pensamiento crítico: La problematización de los contextos en la construcción del conocimiento. Revista Actualidades Pedagógicas, 60, 149-169.

Vieira, R (2018). As comunidades online na promoção do pensamento crítico em didática das ciências. Aveiro: UA Editora.

Whittemore, R, \& Knafl, K (2005). The integrative review: Updated methodology. Journal of Advanced Nursing, 52(5), 546-553. 